\title{
LUNAR TIDAL PHENOMENA AND THE LUNAR RILLE SYSTEM
}

\author{
BARBARA M.MIDDLEHURST
}

Encyclopaedia Britannica, Chicago, U.S.A.

\begin{abstract}
Two types of tide-linked lunar phenomena now exist: the so-called lunar transient events, short-lived changes in brightness or colour and obscurations in small areas of the Moon (the reported duration is typically from a few seconds to a few hours and the areas involved are usually a few kilometres or less in diameter); and the $A$ - and $B$-type seismic signals relayed back from the Apollo 12 passive seismic experiment. The frequency diagrams of both the lunar transien $t$ events and the seismic signals show strong peaks at perigee when the Earth is closest to the Moon, with a smaller peak at apogee (transient events and $B$-type signal only) and both have been attributed to endogenous causes.

Both sets of data appear to be linked to areas where cracks and rilles exist. The most likely interpretation of the two sets of observations is that they are complementary and that some sort of gas release and excitation (in the cases of the glows and colour changes) is involved.

Association of the lunar events with craters with seamed and cracked floors and with other crack systems is described. Only a few associated areas of the Moon-wide lineament systems have been noted, but the Apollo 12 seismometer signals seem likely to originate from the nearby system of parallel features in the Fra Mauro area. The case for possible gas volcanism is examined.
\end{abstract}

\section{Tidal Analysis}

On the Moon, the gravitational tide-raising potential is much simpler than that causing the terrestrial tides. Because of its greater mass, the Earth's contribution dominates; also, the much slower rotation of the Moon and the absence of surface water with the associated tidal lag and distortion allows us to consider body tides under free oscillation only. The terms introduced by the distortion of the Moon's shape are very small and are neglected. However, it is necessary to consider both the changes in distance of the Earth and the librations of the Moon. The radial component of the tidal force at a given point on the Moon, that is, the vertical tidal term, $F_{r}$, in the gravity field can then be expressed in quite simple terms. Neglecting terms in $(r / R)^{2}$ and higher in an expansion in terms of zonal harmonics, we have

$$
\begin{aligned}
F_{r} & =-\frac{G M r}{2 R^{2} R}\left(3 \cos ^{2} z-1\right)+\text { a similar term for the Sun } \\
& =-\frac{G M}{2 R^{2}} \cdot \frac{r}{R}(3 \cos 2 z+1)+\cdots .
\end{aligned}
$$

The solar term is only of the order of $1 \%$ to $3 \%$ of the term due to the Earth but it has been taken into account in the computer-generation of tides undertaken at Houston under the direction of William Chapman of NASA. Results of this programme are used in the following analysis.

In expression (1), the two variables are the Earth's distance and the angular distance, $z$, of the point on the Moon to be considered from the sub-Earth point. The quantity $R$ varies through the anomalistic month but the amount, or amplitude, of this change also changes from month to month over longer cycles due to perturbations of the orbit. The value of $z$ varies because the position of the sub-Earth point varies with the 
librations of the Moon. The value of $F_{r}$ then goes through a modified sinusoidal cycle in the course of an anomalistic month and with passage of time, the amplitudes of the cycle are further modified.

The anomalistic month is on the average $27 \frac{1}{2}$ days, while the tropical month from New Moon to New Moon is $29 \frac{1}{2}$ days, but as Knopoff (1970) has shown, the 6-month perturbation of the lunar orbit by the Sun produces cyclical changes in the length of the anomalistid month; in consequence, perigee is more likely to occur at New or at Full Moon than at the quarter phases. If the observer pattern was such that lunar visual observations tended to take place at New or Full Moon preferentially, then the perigee correlation would have to be adjusted. The pattern of observations up to 1964, used in the frequency diagram for lunar events, did not show such features; most observations are made when shadows are favourable for the enhancement of detail in the object being studied. Except at eclipse times very few observers look at the Moon when it is full and the observations were well distributed with phase. Both the transient events and the seismic $A$ - and $B$-type signals occur most frequently at times of tidal maxima and minima and now appear to originate in similar ways. Of course, no adjustment of the seismic data for phase need be considered.

\section{Characteristics of the Transient Events}

Throughout the lunar literature of the 18th and 19th centuries, with some few reports from earlier sources - the first listed was dated 1540 - descriptions occur of the disappearance of detail for short periods of minutes or hours (clear before and after) and of temporary glows or increases in brightness and colour changes (the so-called 'red spots', 'violet glares' and so on). The best known of modern observations are the reports of colour changes in the lunar crater Alphonsus by N. Kozyrev who took spectra in 1956, the observations of red spots in Aristarchus at Flagstaff, Arizona by the lunar mappers, J. C. Greenacre and E. Barr in October and November, 1963, and the description of a lunar 'volcano' noted by Sir William Herschel on May 4, 1783, which strongly resembled that of the Flagstaff phenomenon except that it was seen on the dark side of the Moon, while Aristarchus was not illuminated by the Sun.

In addition to about 600 visual reports, around 20 records of a more permanent nature, often obtained by chance, are known. The following report was sent to the present author by Dr V. P. Dzhapiashvili of Abastumani Observatory, Georgian S.S.R. in 1970 and has not appeared before in western literature.

Though the phenomenon was registered nearly 19 years ago, the recollection is vivid even today. On the night of July 3,1952, Tamara G. Negrelishvili and I were carrying out a routine programme of electro-polarimetric observations of the Moon with the help of a 33-cm mirror telescope. We were studying the polarization properties of lunar surface formations. The results were published in $1957 \ldots$.

The sky was absolutely clear that night. The author was at the telescope directing it at the object to be measured, guiding and manipulating the analyser.... Reflected light readings for each position of the analyser were repeated to reduce the error of pointing....

We measured two objects - the craters Aristoteles and Eudoxus. It is worth noting that the readings of both series for the respective analyser positions and for each crater separately coincided.

However, when the telescope was directed at the crater Posidonius and when we began to take its 
readings, we witnessed a totally different picture. The pointer of the galvanometer swung to and fro and the readings for the second series of measurements failed to coincide with those of the first. To rule out the possibility of faults in the apparatus we switched back to the craters Aristoteles and Eudoxus. However, we only confirmed our earlier results. We also obtained good results for the crater Aristillus which we observed immediately after the crater Posidonius. So we made the following entry in the record book: "During the observations of the crater Posidonius, the pointer of the galvanometer for some reason swung to and fro. The other craters showed normal readings...." [Later] the author observed Posidonius with the help of the above method in 40 different phases but he failed to witness any similar phenomenon again.

Previous events have been noted as occurring in Posidonius in 1821, 1849, and 1890, and in each case changes in the shadow of the crater were described. Only visual reports were made.

The association of lunar events with perigee and apogee times first led to the conclusion that they were endogenous. This deduction has since been supported by the
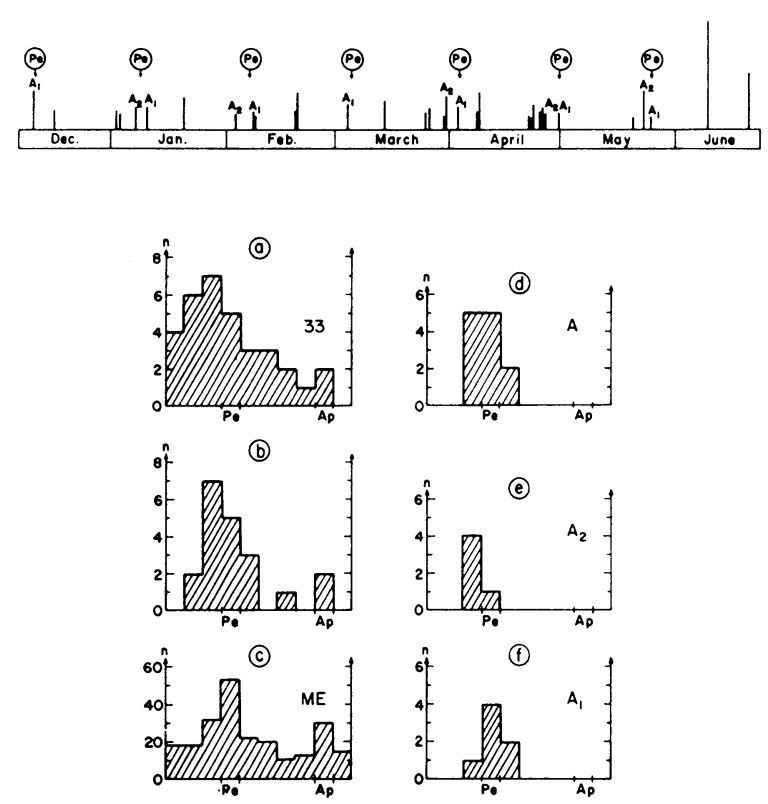

Fig. 1. Frequency distribution for different classes of events with respect to the lunar anomalistic month. (a) 33 of the strongest seismic signals; (b) A- and B-type seismic signals and those with similar frequency spectra; (c) dated lunar transient events before 1964; (d) A-type seismic signals; (e) $A_{1}$-type seismic signals; (f) $\mathrm{A}_{2}$-type seismic signals. Symbols: $\mathrm{Pg}=$ perigee; $\mathrm{Ap}=$ apogee; $\mathrm{ME}=$ Middlehurst Effect. (Courtesy of R. Meissner.)

similarity of the frequency diagram to that found for the $A$ - and $B$-type Apollo 12 seismic signals (Figure 1) and also by the following characteristics.

The distribution of the event sites over the lunar surface is not random but is associated with the borders of the regular maria, and with types of crater containing extensive crack systems, and/or central peaks; in addition to those mentioned already Taruntius, Sabine, Copernicus and Kepler are examples. Most of the larger ray-craters 
have been reported as sites of activity. With the exception of the ring-plain site such as Plato, also often reported as showing an anomalous appearance, all craters that have been listed as event sites have previously been classified as young formations. Other correlations are with the areas where the 'rolling stones' have been found (these objects appeared in Orbiter photographs at the ends of tracks that seemed to have been produced across the lunar surface by gentle movement of the stones themselves; they are mysterious as, in a few cases, the tracks travel uphill, no motive force has been satisfactorily found and there is no evidence to show how the tracks could have been set in motion). Black-haloed craters, and mascons have both been located in active area.

\section{Tides at Aristarchus and Gassendi}

A correlation of increased activity with increases in the amplitude of local tides was noted at two sites for the period from 1963 and 1968.

The crater Aristarchus, named by Hevelius Mons Porphyrites, the burning mountain or red hill, has, with Schröter's Valley and other features of the immediately area - the Aristarchus Uplift - accounted for fully one-third of all the reports of lunar activity. Nevertheless, the times are not random. The usual perigee peak is shown but, in addition, an increase in reported activity was noted by William Chapman (1967) to have occurred when the local tidal amplitudes (see expression (1)) was greatest. In the last few years, since 1963, it has been possible to compare the changes with those at another crater, Gassendi. In 1966, after a long period of quiescence, Gassendi burst into activity. A whole series of reported colour changes, glows, and obscurations were seen by many observers at this time. This spurt died down in 1967. Aristarchus which had been more active during 1963-64 before the Gassendi phenomena began, decreased its activity during the Gassendi peak period (see Figures 2). When the tides for Gassendi and Aristarchus were computed and compared, it was found that in each case the greatest activity was shown at each site at the times of maximum amplitudes of the local tides. The relative positions of the two craters were such that the tides were out of phase with each other.

Observational selection can probably be ruled out. The general morphology of the two craters is similar. Both are large (Aristarchus about $40 \mathrm{~km}$ and Gassendi about $120 \mathrm{~km}$ in diameter) and easily distinguished. The floors of each are seamed with cracks and both have central peaks. Aristarchus, though smaller is brighter. Sunrise occurs one day earlier at Gassendi (10 days after New Moon) than at Aristarchus, and both craters are at about the same distance from the mean centre of the Moon's disc, so that the range and mean value of the tidal amplitudes is the same. Any knowledge of the present analysis among the observers that might have led, even unconsciously, to bias in the observations can be ruled out as the analysis for Gassendi was not begun until most of the data used had been assembled. It is also of interest that an infrared survey made of the lunar surface during a lunar eclipse in 1967 by Salisbury et al. confirmed an earlier survey by Saari and Shorthill in 1964 but noted one additional infrared anomaly or hot spot in Gassendi. 


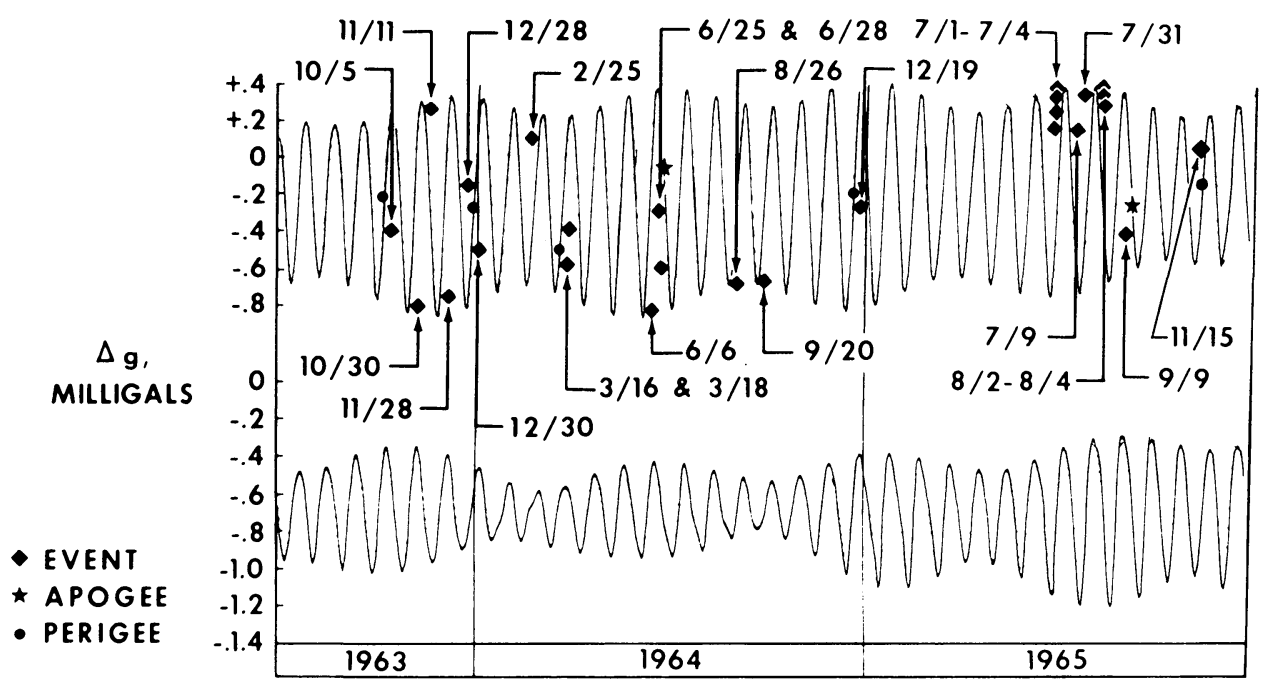

Fig. 2a.

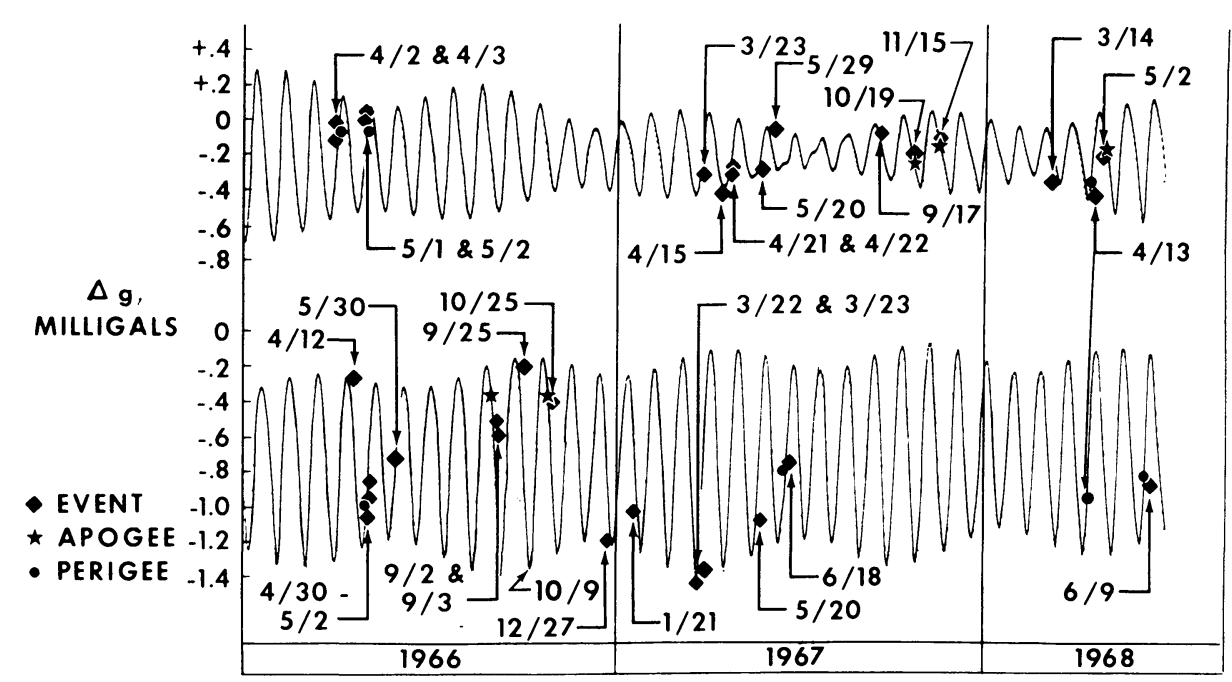

Fig. 2b.

Fig. 2a-b. Tidal-Gravity change. Local tide-raising vertical force change per gram of lunar material (a) for July 1963 to December 1965 and (b) for January 1966 to June 1968. Upper tides at Aristarchus and lower tides at Gassendi. Events at local Aristarchus tidal-gravity values for given date when observed. Each apparent event triggering perigee and apogee is indicated.

\section{The Passive Seismic Experiment of Apollo 12}

With the exception of Aristarchus and its environs, activity in most sites has been sporadic. The passive seismic experiment of Apollo 12 has produced continuous records of moonquakes and meteorite and other impacts since November 19, 1969 up to 


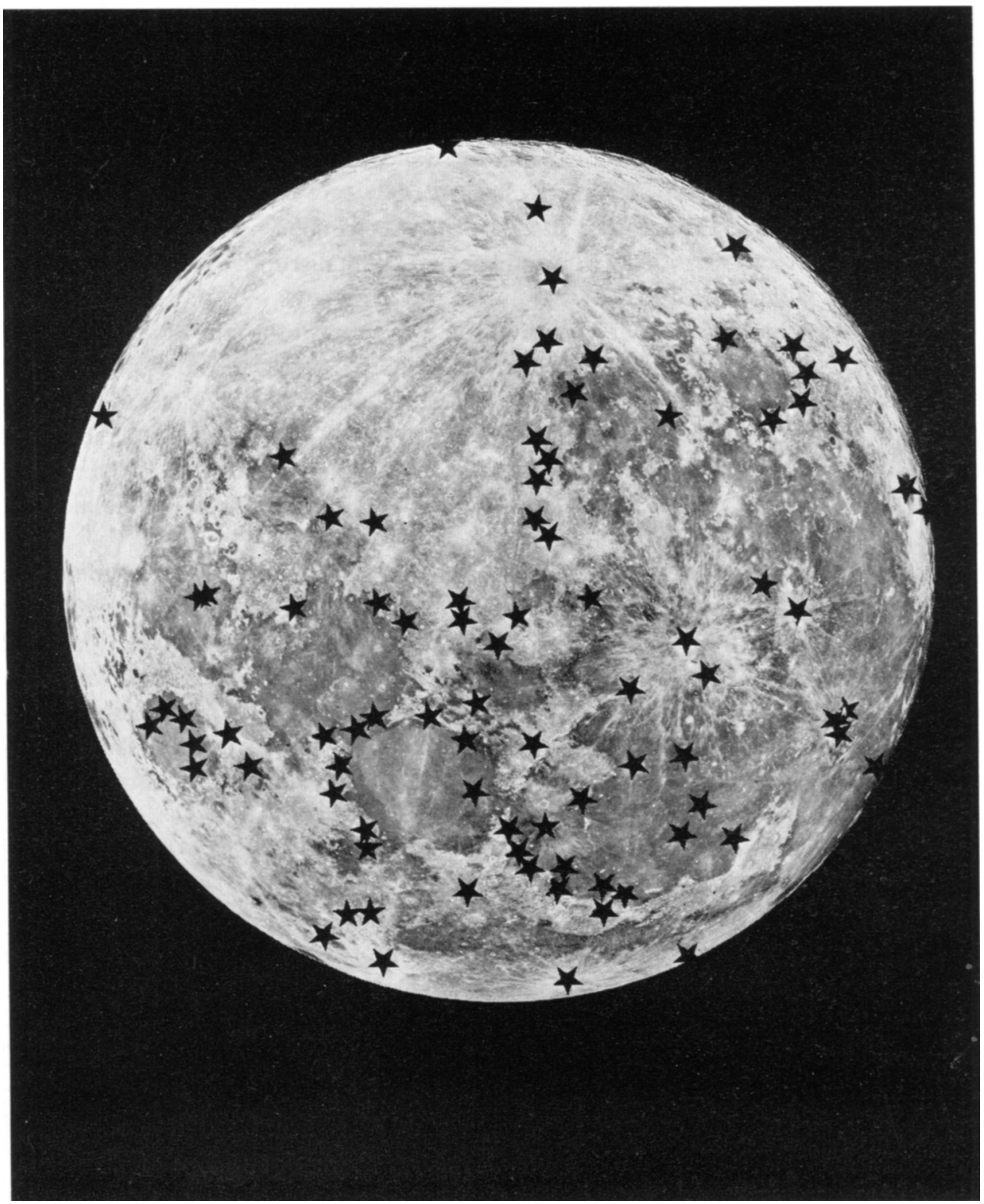

Fig. 3. Sites of lunar transient events for dates before June, 1964. Note that the sites are not distributed at random but occur preferentially around the regular maria, in ray craters, and down the central meridian following the Alphonsus crater chain. 
August 1970. During that time, 208 events, or 23 per month were recorded. The moonquakes are strongly concentrated near perigee and less strongly, as for the transient changes, near apogee. The region of greatest activity has been tentatively located in the vicinity of the well developed set of parallel rilles and lineaments near Fra Mauro and Parry. Figure 1 shows the distribution over the orbit for the first 6 months.

These rilles are directed preferentially from $\mathrm{S} 60^{\circ} \mathrm{W}$ to $\mathrm{N} 60^{\circ} \mathrm{E}$ and at the time of the records were directed towards the sub-Earth point. At such periods, the horizontal component is directed across the rille and has maximum disruptive force. The times of perigee occur within one day of tidal minimum gravity.

Mr Chapman has run tides at Fra Mauro and has shown that the repetitive moonquakes occur at local minimum tidal gravity. Tidal triggering of moonquakes or of transient events might be explained by the release of subsurface fluids as has been previously suggested for the transients alons by Green (1965) and by Middlehurst (1966). Periods of minimum gravity offer periods of decompression and the widest opening of pore spaces when the release of gases is most likely, all other factors for the moment being considered equal.

What is somewhat puzzling is that successive perigees do not correspond to exactly Earth-Moon distances so that a simple correlation with the tidal gravity change is not the whole explanation. In other words, events tend to occur at successive perigees or at successive minima, not at successive times of equal tidal force. This is apparently true of the moonquakes, just as it was earlier of the transient events. More deepseated disturbances, possibly affecting large areas of the Moon may be involved. A second possibility is that either rates of change of stress, or cumulative effects are more potent in triggering off the events and these possibilities are being investigated.

\section{The Lineament System and Other Lunar Rilles and Faults}

In addition to the lunar craters, other features are of structural importance. Three moon-wide systems of lineaments were investigated and plotted by Fielder (1963) and by Strom (1964). They probably represent surface expression of very deep-seated disturbance of the lunar material. The general directions of these lineament systems, which include straight rilles, graben, wrinkle ridges, sections of (polygonal) crater walls and crater chains, are N-S, ENE-WSW, and WNW-ESE, and they continue on the far side of the Moon.

Not many transients are associated with these systems. An apparent fault, the Straight Wall runs N-S near the border of Mare Nubium and is the site of an event reported for 1956 (an obscuration). A N-S chain of craters runs from W. Herschel through Alphonsus to Clavius, along which many events have been reported; this area is apparently depressed below the surrounding level.

Sinuous rilles, of which examples are Schröter's Valley, the Hadley Rille and the Hyginus Rille, all of which are event sites, occur in many areas and may be associated with fluid flow. Schröter's Valley has features strongly resembling terrestrial river meanders and appears to originate in Herodotus, but there are many puzzling features 
in this area and the Orbiter photographs unhappily do not show the whole valley. The Hadley Rille has been compared to a collapsed lava tube. The Hyginus rille has crater chains along it and these seem to be structurally related to it, but clearly have nothing to do with meteorite impact.

No reports of transient events are known for the Fra Mauro-Parry area but the crater forms are quite similar to others that have been reported as sites.

\section{Gas Volcanism}

Many of the processes that lead to volcanism on the Earth are still imperfectly understood. The lunar environment is, however, so different that in any case, it seems unlikely that the phenomena of terrestrial volcanism would be reproduced there. The seismic traces are reported to be quite similar to those occuring in volcanic-triggered seisms on the Earth and passing through porous lava-type rock. Under external conditions of near-perfect vacuum, many substances, liquid under standard temperatures and pressures, would soon form pockets of gas that could escape through fissures and produce disturbances of the rock detectable as lowlevel seismic signals of the kind recorded by the Apollo 12 seismometer. The transient event descriptions are entirely consistent with this possibility, and the hypothesis has been further strengthened by Dr Mills' elegant production of not one but many types of crater forms by fluidization of dust through the passage of gas through it in the laboratory. See chapter by Mills, p. 407.)

In summary, The mechanism of production of both the $A$ - and $B$-type seismic traces and the transient events is likely to be tide-triggered disturbance of existing cracks, not necessarily in progressive faulting, but by repeated opening and closing of the pores so that gas within the Moon can alternately be built up and released.

\section{References}

Burley, J. M. and Middlehurst, B. M.: 1966, Proc. Nat. Acad. Sci. 55, 1007.

Chapman, W. B.: 1967, Geophys. Res. 72, 6293.

Chapman, W. B. and Middlehurst, B. M.: 1971, (abstract), Trans. Am. Geophys. Union 52, 4. Chapman, W. B. and Middlehurst, B. M.: 1972, (abstract), Trans. Am. Geophys. Union 53, 4.

Fielder, G.: 1963, Quart. J. Geol. Soc. London, No. 119, pp. 65.

Green, J.: 1965, Ann. N.Y. Acad. Sci. 123, 433.

Knopoff, L.: 1970, Moon 2, 143.

Latham, G. and Meissner, R., et al.: NASA Prelim. Sci. Rep. for Apollo 12 (SP 235).

Meissner, R., Sutton, G., and Duennebier, F.: 1970, Mondbeben, Umschau, Heft 4, 111.

Middlehurst, B. M.: 1967, Rev. Geophys. 5, 173.

Middlehurst, B. M. and Chapman, W. B.: 1971, Strolling Astronomer 23, 17.

Strom, R.: 1964, Comm. Lunar Planet. Lab. 2, 205. 